\title{
Hypothalamic MCH Neuron Activity Dynamics during Cataplexy of Narcolepsy
}

Ying Sun and $\mathbb{D}^{M}$ Meng Liu

https://doi.org/10.1523/ENEURO.0017-20.2020

Department of Psychiatry and Behavioral Sciences, Medical University of South Carolina, Charleston, SC 29425

\section{Visual Abstract}
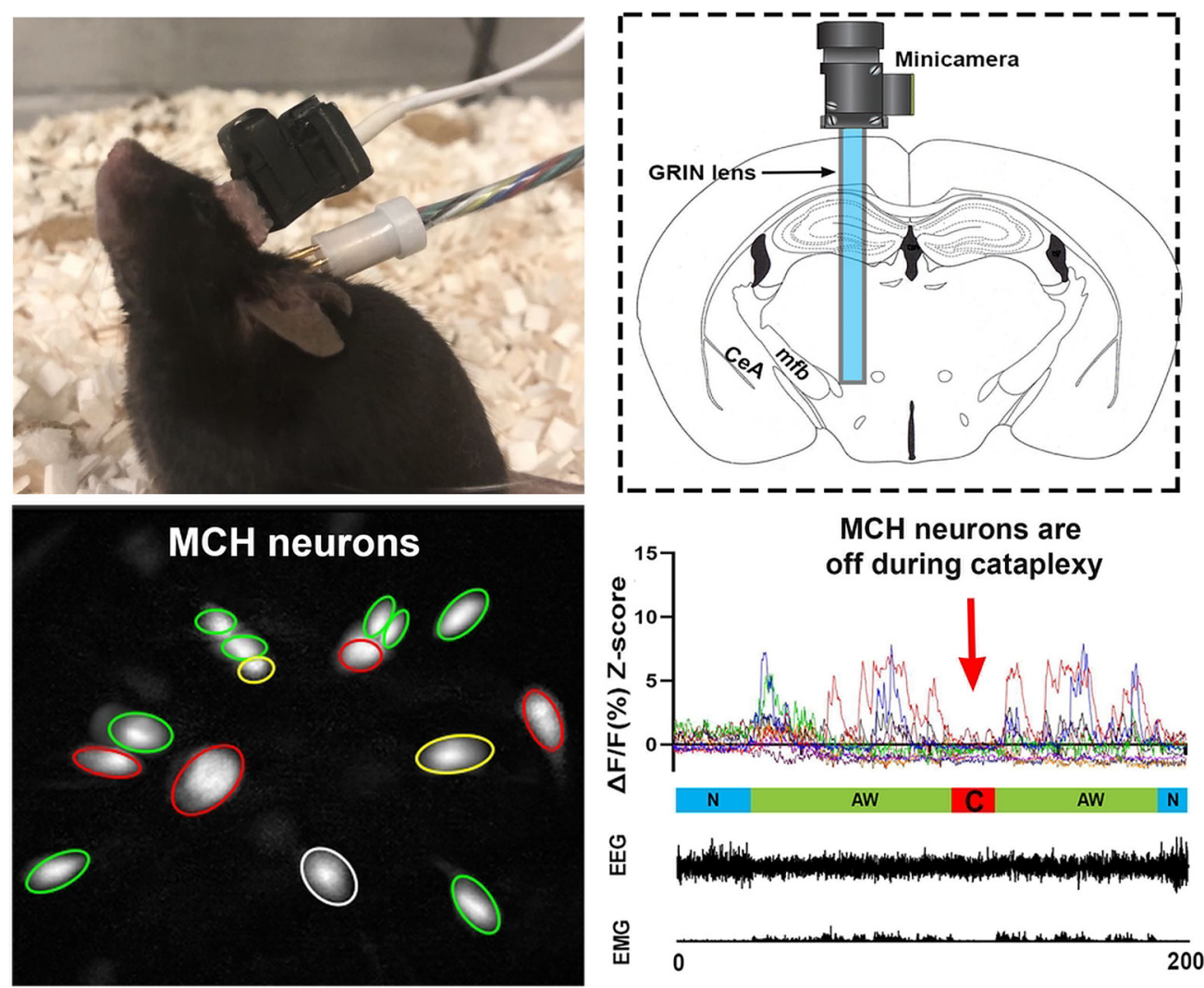

\section{Significance Statement}

The cataplexy of narcolepsy shares many similarities with normal rapid eye movement (REM) sleep. Thus, REM sleep-promoting melanin-concentrating hormone $(\mathrm{MCH})$ neurons are thought to be part of the cataplexy circuitry. Suppressing $\mathrm{MCH}$ neurons is assumed to block cataplexy. However, we found that $\mathrm{MCH}$ neurons were always inactive during cataplexy in orexin knock-out mice. Pre-cataplexy activations of $\mathrm{MCH}$ neurons were no different from the activations during regular waking episodes. Importantly, cataplexy could occur in the absence of these pre-cataplexy activations. These results suggest that $\mathrm{MCH}$ neurons are not a key component of the cataplexy circuitry in orexin knock-out mice. 
Hypothalamic orexin (hypocretin, HCRT) deficiency causes sleep disorder narcolepsy with cataplexy in humans and murine. As another integral group of sleep/wake-regulating neurons in the same brain area, the melanin-concentrating hormone $(\mathrm{MCH})$ neurons' involvement in cataplexy remains ambiguous. Here we used the live animal deep-brain calcium $\left(\mathrm{Ca}^{2+}\right)$ imaging tool to record $\mathrm{MCH}$ neuron dynamics during cataplexy by expressing calcium sensor GCaMP6s into genetically defined $\mathrm{MCH}$ neurons in orexin knock-out mice, which are a model of human narcolepsy. Similar to wild-type mice, $\mathrm{MCH}$ neurons of the narcoleptic mice displayed significantly higher $\mathrm{Ca}^{2+}$ transient fluorescent intensity during rapid eye movement (REM) sleep and active waking (AW) episodes compared with non-REM (NREM) sleep. Moreover, $\mathrm{MCH}$ neurons displayed significantly lower $\mathrm{Ca}^{2+}$ signals during cataplexy. Importantly, a pre-cataplexy elevation of $\mathrm{Ca}^{2+}$ signals from $\mathrm{MCH}$ neurons was not a prerequisite for cataplexy initiation. Our results demonstrated the inactivation status of $\mathrm{MCH}$ neurons during cataplexy and suggested that $\mathrm{MCH}$ neurons are not involved in the initiation and maintenance of cataplexy in orexin knock-out mice.

Key words: cataplexy; MCH; narcolepsy; sleep

\section{Introduction}

Orexin (hypocretin, HCRT) and melanin-concentrating hormone $(\mathrm{MCH})$ are hypothalamic neuropeptides regulating sleep and wakefulness. Orexin neurons are wake-active, promoting arousal and maintaining wakefulness (Adamantidis et al., 2007; Alexandre et al., 2013) while $\mathrm{MCH}$ neurons are predominantly rapid eye movement (REM) sleep-active and promote REM sleep (Jego et al., 2013; Konadhode et al., 2013; Blanco-Centurion et al., 2016, 2019; Izawa et al., 2019). Lack of orexin neurons or loss of the orexin gene causes sleep disorder narcolepsy and its signature symptom cataplexy, a sudden skeletal muscle atonia during waking (Chemelli et al., 1999; Lin et al., 1999; Hungs and Mignot, 2001). Cataplexy or cataplexy-like behavior has not been reported in $\mathrm{MCH}$ or its receptor knock-out mice, suggesting that there is no causal effect between $\mathrm{MCH}$ deficiency and cataplexy. However, whether $\mathrm{MCH}$ neurons play a role in cataplexy in narcolepsy patients or narcoleptic animals is still unknown. The question could be answered by manipulating $\mathrm{MCH}$ neurons in narcoleptic animals using optogenetic or chemogenetic tools. Indeed, a recent study indicated that chemogenetically activating $\mathrm{MCH}$ neurons increased cataplexy and abnormal REM sleep in orexin knock-out mice (Naganuma et al., 2018). However, we believe that the instinctive status of $\mathrm{MCH}$ neurons during cataplexy is still crucial for understanding the exact involvement of $\mathrm{MCH}$ neurons in cataplexy and for designing optimal intervention

Received January 20, 2020; accepted March 7, 2020; First published April 17, 2020.

The authors declare no competing financial interests.

Author contributions: M.L. designed research; Y.S. and M.L. performed research; Y.S. and M.L. analyzed data; M.L. wrote the paper.

This work was supported by National Institutes of Health Grants 1R01NS096151 and 1R21NS101469.

Acknowledgment: We thank Dr. Priyattam Shiromani, Dr. Blanco-Centurion Carlos, Dr. SiWei Luo, and Aurelio Vidal-Ortiz at the Medical University of South Carolina for advice, guidance, and technical support on animal surgery and calcium imaging data processing. We also thank Dr. Jiexiang $\mathrm{Li}$ at the College of Charleston for advice on data processing.

Correspondence should be addressed to Meng Liu at liumen@musc.edu.

https://doi.org/10.1523/ENEURO.0017-20.2020

Copyright $@ 2020$ Sun and Liu

This is an open-access article distributed under the terms of the Creative Commons Attribution 4.0 International license, which permits unrestricted use, distribution and reproduction in any medium provided that the original work is properly attributed. strategies to block cataplexy. For instance, if $\mathrm{MCH}$ neurons are active during cataplexy, further stimulation/excitation may have no noticeable effects due to the "ceiling" effect. Likewise, further inhibition might be ineffective due to the "floor" effect if $\mathrm{MCH}$ neurons are already silent during cataplexy. In this study, we took advantage of the novel genetic $\mathrm{Ca}^{2+}$ imaging tool to record real-time $\mathrm{MCH}$ neuronal activities during spontaneous cataplexy and emotional cataplexy induced by either positive (palatable food: milk) or negative (innate fear: predator odor) emotions. We want to know whether and how $\mathrm{MCH}$ neurons contribute to the generation or propagation of cataplexy.

\section{Materials and Methods}

\section{Animals and surgery}

All the manipulations done to the mice followed the policies established in the National Institutes of Health Guide for the Care and Use of Laboratory Animals and were approved by the Institutional Animal Care and Use Committee (protocol \#IACUC-2019-00,723).

To specifically target $\mathrm{MCH}$ neurons in narcoleptic mice, orexin knock-out mice $\left(\mathrm{Hcrt}^{-1-}\right)$ mice (derived from founders donated by Yanagisawa, Southwestern Medical Center, Dallas, TX) were crossed with $\mathrm{MCH}$-Cre mice (The Jackson Laboratory; stock \#014099). Offspring with the confirmed genotype $\mathrm{MCH}-\mathrm{Cre}^{+/-} / \mathrm{Hcrt}^{-/-}$were used as the narcoleptic group $(n=8$, both sexes, $6-10$ months of age). Genotype validation on mice tail snips was done offsite by Transnetyx. The temperature in the mice housing/ recording room was always maintained at $23-25^{\circ} \mathrm{C}$ under a 12/12 h light/dark cycle (lights on at 6 A.M.). Mice were given ad libitum access to regular laboratory food and water.

Under deep anesthesia (isoflurane 1.0-2.0\%) and using a stereotaxic frame (Kopf), adeno-associated virus (AAV) vectors with Cre-inducible expression of GCaMP6 slow (AAV5-CAG-DIO-GCaMP6s, Titer: $3.48 \times 10^{13}$ genomic copies/ml; University of Pennsylvania Preclinical Vector Core) were microinjected unilaterally into the lateral hypothalamus at the following coordinates: $1.11 \mathrm{~mm}$ posterior to bregma, $1.25 \mathrm{~mm}$ lateral to the sagittal suture, and 4.60 $\mathrm{mm}$ ventral to the brain surface (Hof et al., 2000). Viral vectors were delivered in a volume of $500 \mathrm{nl}$ using a 10.0$\mu \mathrm{l}$ Hamilton syringe coupled to a 33-Gauge stainless steel injector (Plastics One). Injections were done gradually 


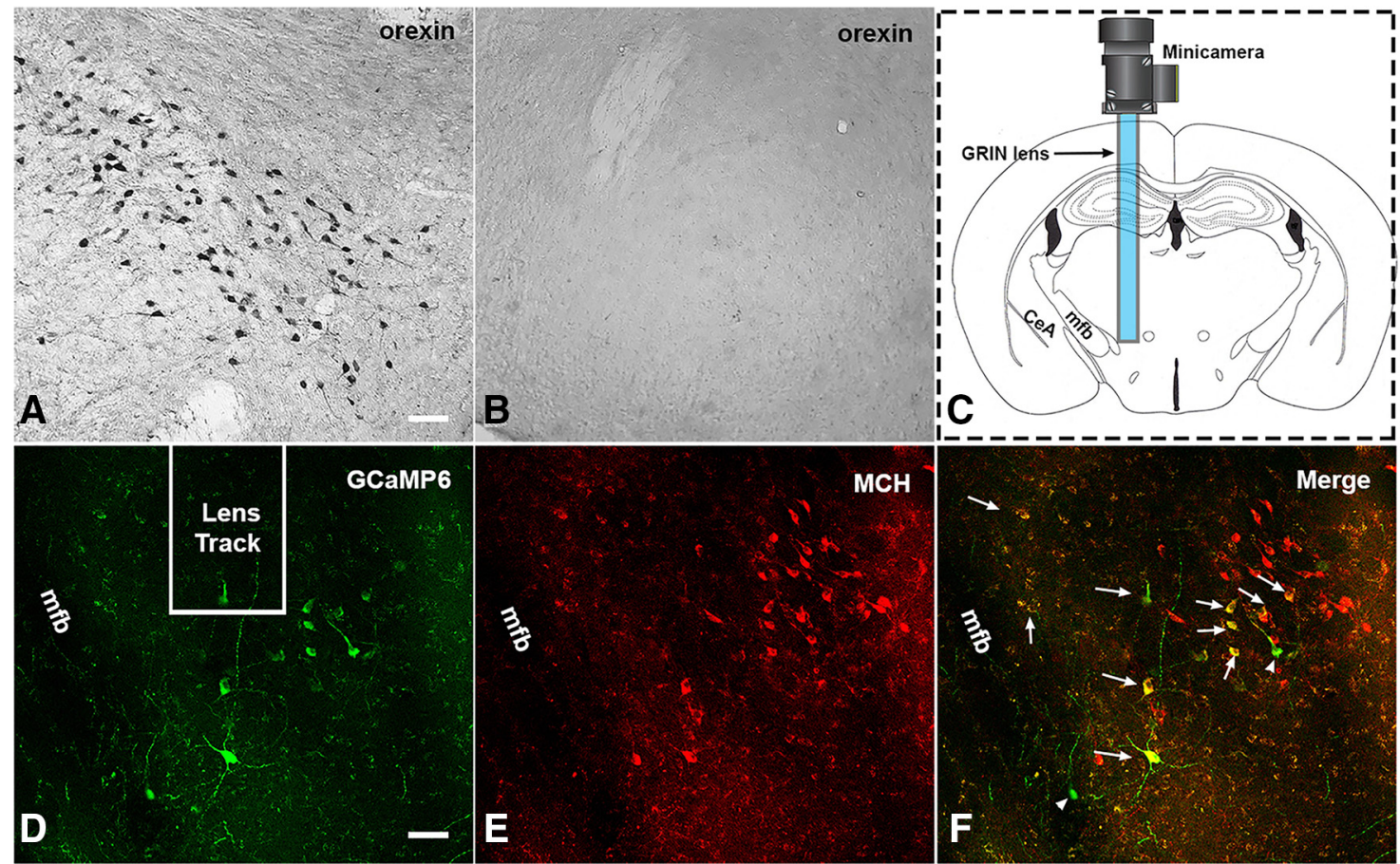

Figure 1. $\mathrm{H}$ istology results. Orexin ${ }^{+}$neurons were found in the lateral hypothalamus of the wild-type $\mathrm{MCH}-\mathrm{Cre}$ mice $(\boldsymbol{A})$ but were absent in the $\mathrm{MCH}-\mathrm{Cre}^{+/-} / \mathrm{HCRT}^{-1-}$ narcoleptic mice $(\boldsymbol{B}) . \boldsymbol{C}$, Illustration of the GRIN lens and miniature camera installation. $\boldsymbol{D}-\boldsymbol{F}$, GCaMP6s and $\mathrm{MCH}$ expression in the hypothalamus near the end of the lens. Long arrows, GCaMP $6 \mathrm{~s}^{+} / \mathrm{MCH}^{+}$double-labeled neurons. Short arrowhead, A GCaMP6s ${ }^{+} / \mathrm{MCH}^{-}$neuron. CeA, the central nucleus of the amygdala; $\mathrm{mfb}$, medial forebrain bundle. Scale bar $=50 \mu \mathrm{m}$.

Figure Contributions: Ying Sun performed immunostaining and confocal imaging. Meng Liu produced the montage.

over 25 min. After microinjection, the injector needle was left in place for $15 \mathrm{~min}$ and then withdrawn slowly. At this time, and following the same injection track, a miniature Gradient Refractory INdex lens (GRIN, outer diameter: $0.6 \mathrm{~mm}$, length: $7.3 \mathrm{~mm}$; Inscopix Inc) was driven into the brain just above the injection target and cemented to the skull. Then, as described elsewhere (Liu et al., 2011), four small screw-type electrodes and a pair of plate-type electrodes (Plastics One) were implanted onto the mouse skull and nuchal muscles for recording the electroencephalogram (EEG) and electromyogram (EMG) activity, respectively. Ten days after the GRIN lens placement, mice were deeply anesthetized again (1.0-2.0\% isofluorane). A baseplate was attached to a single photon miniaturized fluorescence microscope/CCD camera (nVoke from Inscopix). The miniaturized microscope, along with the baseplate, were carefully placed atop the GRIN lens. The distance between the miniaturized microscope and the GRIN lens top was precisely adjusted until fluorescent neurons came into focus. At this focal point, the baseplate was secured around the GRIN lens cuff with dental cement, and then the microscope was detached. To protect the GRIN lens from debris and scratches, a cap was secured onto the baseplate. One week later, mice were habituated to the recording experiment setting for three consecutive days before the sleep and $\mathrm{Ca}^{2+}$ recording started.

\section{Sleep recording and identification of sleep states or cataplexy}

After being amplified and filtered $(0.3-100 \mathrm{~Hz}$ for EEG; 100-1000 Hz for EMG, MP150 system; Biopac Systems
Inc.), the EEG/EMG signals were acquired and synchronized to the imaging of the $\mathrm{Ca}^{2+}$ transients. In parallel, a night-vision camera was used to record mouse behavior. Streaming video of the mouse behavior was also synchronized with the imaging of the $\mathrm{Ca}^{2+}$ transients (Neuroscience Studio acquisition software, Doric Lenses Inc). NeuroExplorer software (Nex Technologies) was used to plot the spectrogram of the EEG activity (1-s window size and 0.5 -s overlap).

The EEG/EMG data (as CSV files) along with synchronized behavior video files were then transferred to the SleepSign software (KISSEI Comtec Ltd.) and scored in 4-s epochs as wakefulness, non-REM (NREM) sleep, REM sleep, and cataplexy. Wakefulness was identified by the presence of desynchronized EEG coupled with high amplitude EMG activity. In this study, we focused on active wakefulness (AW) when the mice displayed behaviors such as walking, rearing, grooming, eating, drinking, digging, and exploring. NREM sleep was scored when the EEG showed high-amplitude/low-frequency waves $(\delta$ waves) together with a lower EMG activity relative to waking. REM sleep was identified by the presence of regular EEG $\theta$ activity coupled with very low EMG activity.

To be qualified as a cataplexy attack, an episode had to meet the following criteria: (1) an abrupt episode of nuchal atonia lasting at least $8 \mathrm{~s}$, (2) immobility during the episode, (3) $\theta$ activity dominant EEG during the episode, and (4) at least $40 \mathrm{~s}$ of wakefulness preceding the episode (discrete cataplexy) or the first episode when several 
cataplexy episodes occur sequentially. The above criteria were slightly modified from the International Working Group on Rodent Models of Narcolepsy (Scammell et al., 2009). We also named the 40-s AW episodes preceding the cataplexy as pre-cataplexy AW episodes (Pre-C AW) to distinguish them from other AW episodes not followed by cataplexy (N-C AW).

\section{Miniature microscopy $\mathrm{Ca}^{2+}$ transients imaging}

A t 10 A.M., the mouse was gently restrained (swaddled in Terrycloth), while a dummy miniscope (with the same size and weight as the real miniature fluorescent microscope) was attached to its baseplate. At the same time, a lightweight cable was plugged to record the EEG/EMG signals. The tethered mouse was then returned to the home cage and allowed to adapt for $6 \mathrm{~h}$ for three consecutive days. On the fourth day (the recording day), the same adaptation routine was followed with the real miniscope, but at 5 P.M., $\mathrm{Ca}^{2+}$ transients-derived fluorescence began to be imaged by the nVoke miniaturized microscope/CCD camera (Inscopix) and collected by its acquisition software. $\mathrm{Ca}^{2+}$ associated fluorescence was continuously generated by a blue LED (power: $0.2 \mathrm{~mW}$ ) and imaged at five frames per second (fps). To synchronize the timestamps of the $\mathrm{Ca}^{2+}$ imaging with the EEG/EMG, a $T \mathrm{TL}$ signal was sent from the nVoke interface console into the Doric console. After $30 \mathrm{~min}$ of undisturbed recording, the mouse was exposed to milk (whole milk, $2 \mathrm{ml}$ ) for $30 \mathrm{~min}$ and coyote urine (1 ml, PredatorPee) for $30 \mathrm{~min}$ between 5:30 and 7 P.M. (Liu et al., 2016).

\section{Analysis of $\mathrm{Ca}^{2+}$ transients imaging data}

The $\mathrm{Ca}^{2+}$ transient data were processed offline by the Inscopix data processing software (version 1.1.2). Briefly, raw movies were first pre-processed to correct for defective pixilation, row noise, and dropped frames. Preprocessed movies were then corrected for motion artifacts to generate the steadiest $\mathrm{Ca}^{2+}$ fluorescent signals. The motion-corrected movies were subsequently mean filtered. To normalize the $\mathrm{Ca}^{2+}$ signals, a single frame average projection of the filtered movie was generated. The average frame was used as the background fluorescence (F0) to calculate the instantaneous normalized $\mathrm{Ca}^{2+}$ fluorescent signals $(\Delta \mathrm{F} / \mathrm{F})$ according to the formula; $(\Delta F / F)_{i}=F_{i}-F O / F 0$ where i represents each movie frame. The normalized movie or " $\Delta F / F$ movie" was then used for semiautomatic extraction of $\mathrm{Ca}^{2+}$ fluorescent signals associated with individual cells based on the principal and independent component analysis (PCAICA). Regions of interest (ROIs) identified by the PCA-ICA were visually selected as candidate cells based on $\triangle \mathrm{F} / \mathrm{F}$ and the image (cell-morphology). To be chosen as bona fide neurons, $\mathrm{Ca}^{2+}$ traces had to fulfill the canonic $\mathrm{Ca}^{2+}$ spike waveform featuring fast-rising onset followed by slower decaying signals. The $\mathrm{Ca}^{2+}$ traces $(\Delta \mathrm{F} / \mathrm{F})$ of each $\mathrm{ROI}$ (cell) were further standardized as Z-scores using the mean and $\mathrm{SD}$ of each cell's $\Delta \mathrm{F} / \mathrm{F}$ [Z-score $=(\Delta \mathrm{F} / \mathrm{F}-$ mean $) / \mathrm{SD}]$. Since the lowest Z-score values in the normal sleep/wake states during the undisturbed recordings were observed in NREM sleep, and no cell reached its maximal activity during NREM sleep, we used the average Z-score of the NREM sleep
(Z-NREM) as the baseline. If the average Z-score of a cell during a specific state is equal to or greater than (Z-NREM +1.0) during the undisturbed recording period, it was scored as an "ON" cell in that state. We then used the percentage threshold, which was defined as $80 \%$ of the maximum Zscore value during the whole recording period, to detect the frequency of the prominent neuronal peak events (per cell, per minute), and we set $2 \mathrm{~s}$ as the minimum interval between two adjacent peaks.

\section{Histology}

At the end of the study, the mice were anesthetized with isoflurane $(5 \%)$ and perfused transcardially with $0.9 \%$ saline (5-10 ml) followed by $10 \%$ buffered formalin in $0.1 \mathrm{M} \mathrm{PBS} \mathrm{(50}$ $\mathrm{ml})$. Mice brains were harvested and cross-sectioned at 40$\mu \mathrm{m}$ thickness (four sets) on a compresstome (Precisionary Instruments). To visualize the GRIN lens track and the location of the GCaMP6s transgene expression, coronal sections were scanned on a Leica fluorescent microscope. To verify that GCaMP6s was expressed exclusively in $\mathrm{MCH}$ neurons, $\mathrm{MCH}$ immunostaining was performed on one set of brain sections. Briefly, the sections were incubated at room temperature for $24 \mathrm{~h}$ with rabbit anti-MCH polyclonal antibody (1:500 dilutions, Phoenix Pharmaceuticals), followed by 1-h incubation with Alexa Fluor 568 donkey anti-rabbit lgG (1:500). GCaMP6s and MCH-positive cells were counted on digitized images. To confirm the correctness of the genotyping, orexin immunostaining was conducted on a separate set of brain sections. Briefly, the sections were incubated at room temperature for $24 \mathrm{~h}$ with goat anti-orexin polyclonal antibody (1:5000 dilutions, Santa Cruz Biotechnology), followed by 1-h incubation with biotinylated donkey anti-goat $\lg G$ (1:500, Millipore) secondary antibody and finally labeled using $A B C-D A B-$ nickel staining (Vector Laboratories).

\section{Statistical analysis}

The one-way ANOVA and Bonferroni post hoc tests (SPSS, version 25) were used to compare the means of the Z-score values among the sleep/wake states. Statistical significance was evaluated at the $p<0.05$ (two-tailed) level (Kirk, 1968).

\section{Results}

\section{$\mathrm{MCH}$ /orexin immunostaining and GCaMP6s expression}

We found that orexin ${ }^{+}$neurons were utterly absent in the $\mathrm{MCH}-\mathrm{Cre}^{+/-} / \mathrm{Hcrt}^{-/-}$mice used in this study, unlike the dense distributions of orexin ${ }^{+}$neurons in the lateral hypothalamus of the wild-type $\mathrm{MCH}$-Cre mice (Fig. $1 A, B$ ). These immunostaining results, together with the genotyping results and signature cataplexy behaviors, validated the animal model of narcolepsy. We recorded continuous $\mathrm{Ca}^{2+}$ transient signals from four of the eight mice installed with the GRIN lenses. In these four mice, GCaMP6s predominantly expressed within the lateral hypothalamus area (Fig. 1C,D). The MCH immunostaining results showed that $\sim 95-98 \%$ of the GCaMP6s expressing neurons also contained $\mathrm{MCH}$ in the cytoplasm (Fig. 1,D-F). 

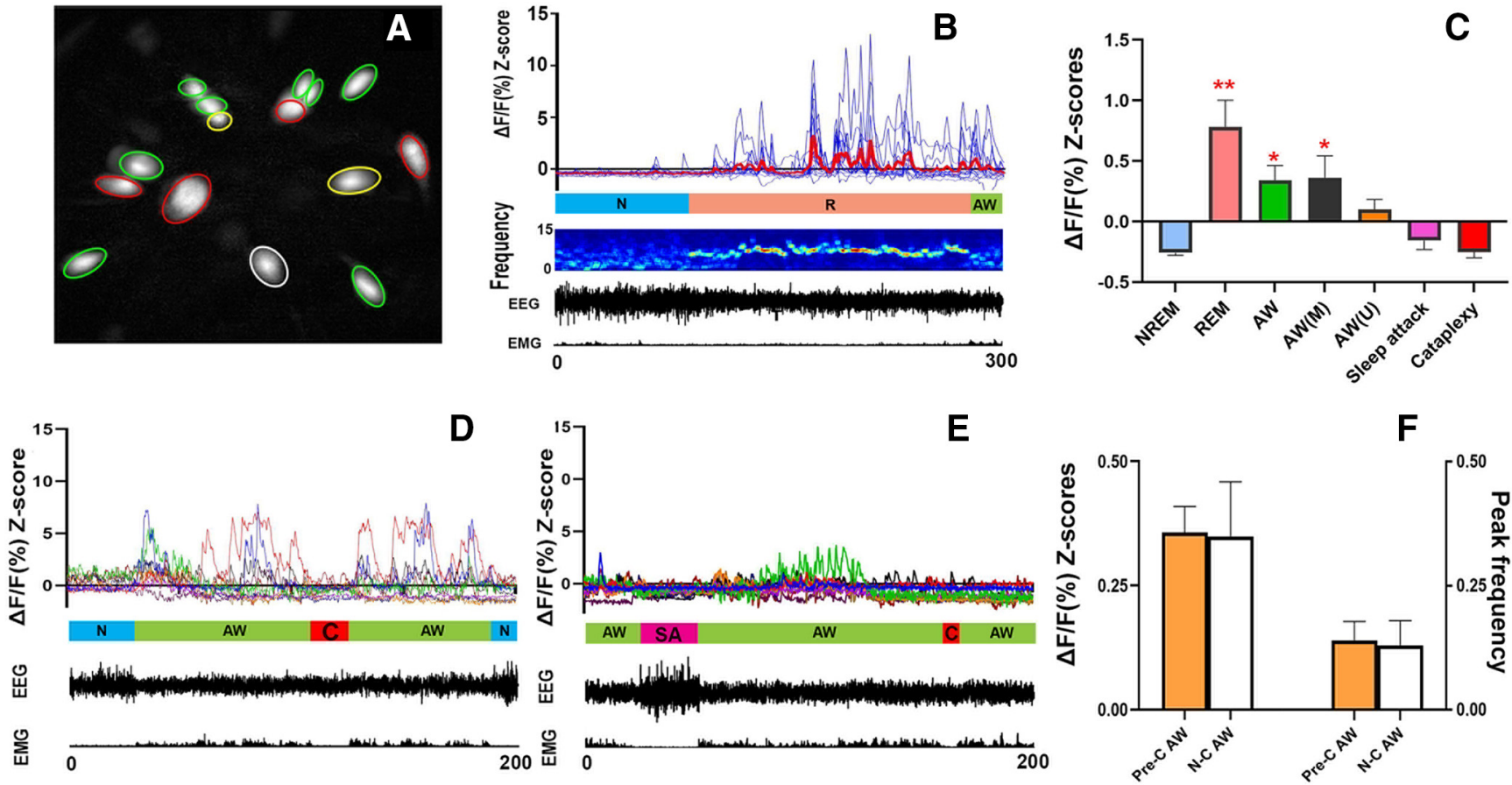

Figure 2. $\mathrm{Ca}^{2+}$ imaging/transient and synchronized behavioral data. $\boldsymbol{A}$, Map of 15 cells recorded from Mouse MLCH02. REM-ON cells are green circled, AW-ON cells are red circled, REM/AW-ON cells are yellow circled, and an unscored cell is white circled. $\boldsymbol{B}$, Plots of $\mathrm{Ca}^{2+}$ transient of the cells from $\boldsymbol{A}$ (thicker red line represents the average), followed by mouse EEG power spectrogram (1-s window size and 0.5-s overlap), EEG/EMG waveforms. N, NREM sleep; R, REM sleep; AW, AW. C, Average Ca ${ }^{2+}$ transient signal intensity ( $\triangle \mathrm{F} / \mathrm{F}$ Z-scores) of $\mathrm{MCH}$ neurons in various states. AW $(M)$, AW during milk exposure; AW ( $U$ ), AW during coyote urine exposure; $* * p<0.001$ or $* p<0.01$ compared with NREM sleep, sleep attack, and cataplexy. $\boldsymbol{D}, \boldsymbol{E}, \mathrm{Ca}^{2+}$ transients and EEG/EMG waveforms from mouse MLCH03 after milk exposure $(\boldsymbol{D})$ and predator odor exposure $(\boldsymbol{E})$. C, cataplexy; SA, sleep attack. $\boldsymbol{F}$, No significant differences in $\mathrm{Ca}^{2+}$ signal intensities (Z-scores) and peak frequencies (peaks/cell/min) between Pre-C AW episodes and NC AW episodes were found.

Figure Contributions: Ying Sun and Meng Liu performed data analysis. Meng Liu produced the montage.

\section{MCH neuronal activities during undisturbed sleep/ wake cycle}

Altogether, we imaged $67 \mathrm{MCH}$ neurons from four narcoleptic mice (two males and two females). These $\mathrm{MCH}$

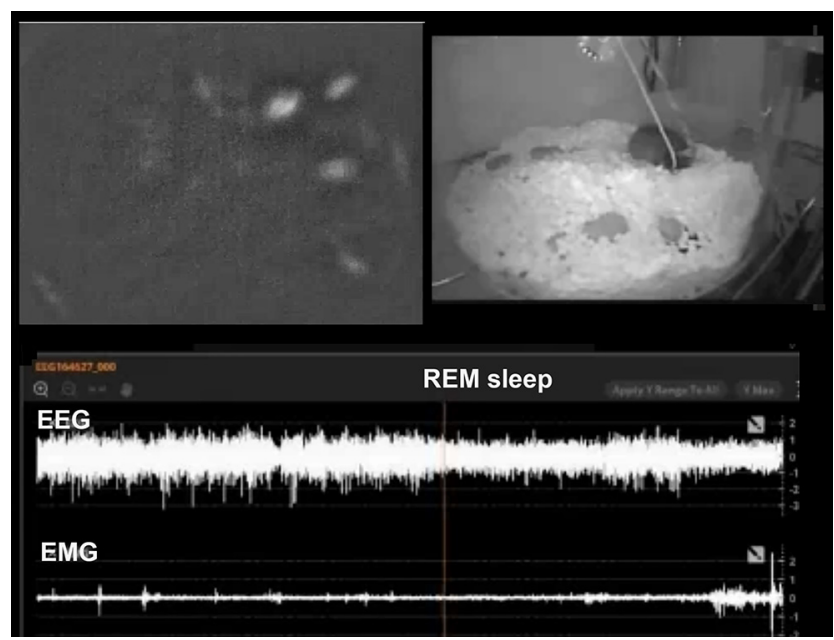

Movie 1. The raw calcium movies with synchronized EEG/EMG and animal behavior video during the undisturbed sleep recording shown in Figure $2 B$ (played at $8 x$ speed). [View online] neurons displayed activity patterns very similar to those of the wild-type $\mathrm{MCH}$ neurons (Blanco-Centurion et al., 2019; Fig. 2A,B; Movie 1). A marked increase in the average Z-scores was observed during REM sleep and AW when compared with NREM sleep (Fig. 2C). The results of

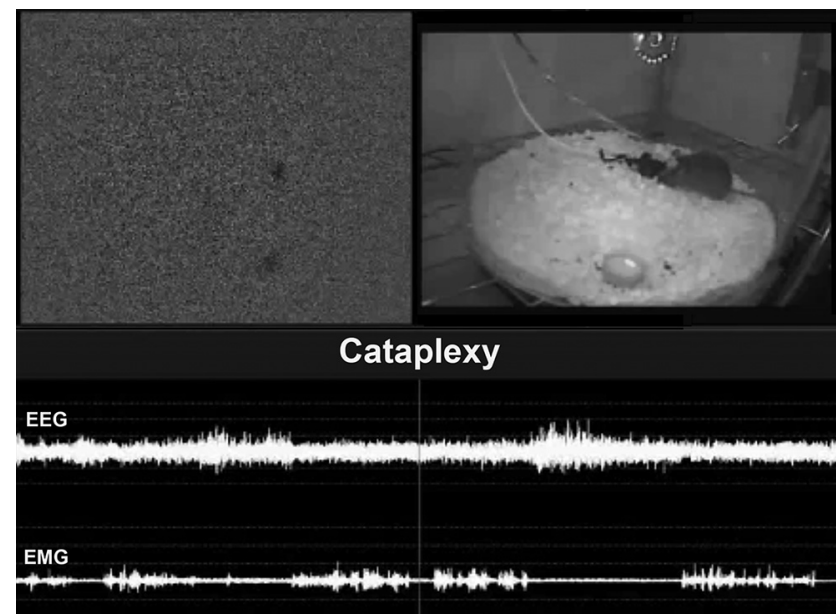

Movie 2. The raw calcium movies with synchronized EEG/EMG and animal behavior video during milk exploring and drinking shown in Figure 2D (played at 8x speed). [View online] 


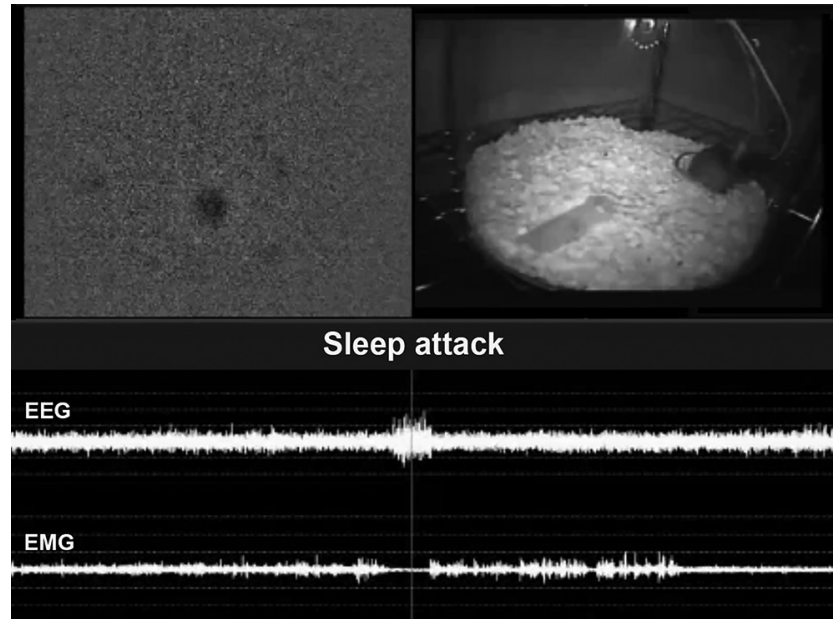

Movie 3. The raw calcium movies with synchronized EEG/EMG and animal behavior video during predator odor exposure shown in Figure 2E (played at 8x speed). [View online]

the one-way ANOVA revealed a significant effect on the $\mathrm{Ca}^{2+}$ fluorescent intensity $\Delta \mathrm{F} / \mathrm{F}$ Z-scores $\left(F_{(6,468)}=58.38\right.$, $p<0.001)$ depending on individual sleep/wake states. The cells were categorized as ON (activated) cells based on the $\mathrm{Ca}^{2+}$ fluorescent intensity data before exposures to milk or coyote urine. Of the total 67 cells, $59.70 \%$ (40/ 67) were REM-ON only, $20.90 \%$ (14/67) were AW-ON only, $14.93 \%$ (10/67) were REM/AW-ON, and 4.48\% (3/ 67) were unscored (no significant activity change among the different sleep/wake states was found during the undisturbed recording).

\section{$\mathrm{MCH}$ neuronal activities during cataplexy and sleep attack}

In addition to cataplexy, some narcoleptic mice may present another behavioral arrest called sleep attack, which is a brief NREM sleep intrusion during AW such as exploring, grooming, and eating. Sleep attacks were characterized by a mixture of $\delta$ and $\theta$ activities in the EEG, in contrast to cataplexy where $\theta$ activity dominates. We recorded 12 cataplexy bouts (three spontaneous ones before any exposure, four after the milk exposure, and five after the odor exposure) and eight sleep attack bouts (four before any exposure, two after the milk exposure, and two after the odor exposure) during the whole 2-h recording periods. The average $\mathrm{Ca}^{2+}$ intensity levels during cataplexy and sleep attack were similar to those of the NREM sleep and significantly lower than that of the REM sleep and AW. Importantly, neither "cataplexy-ON" nor "sleep attack-ON" cells were identified during the whole 2-h recording (Fig. 2D,E; Movie 2, Movie 3).
In the $30 \mathrm{~min}$ of undisturbed (before any exposure) and milk exposure recording, significantly elevated $\mathrm{Ca}^{2+}$ signal intensities from $\mathrm{MCH}$ neurons could be observed during $\mathrm{N}-\mathrm{C}$ AW as well as AW episodes closely followed by a cataplexy bout (we named these episodes as Pre-C AW). The increased $\mathrm{Ca}^{2+}$ signal during these Pre-C AW suddenly dropped to the basal level once the cataplexy began (Fig. 2D,E). Upon the predator odor (coyote urine) exposure, the mice showed several typical behavioral signs of fear, including exploration, avoidance, and escape attempts. However, the $\mathrm{Ca}^{2+}$ signal intensities from $\mathrm{MCH}$ neurons showed insignificant increases during these fear response behaviors. In the meantime, sleep attack or cataplexy continued to be observed, during which time $\mathrm{MCH}$ neurons continued to display low $\mathrm{Ca}^{2+}$ fluorescent intensities (Fig. 2E; Movie 3).

To better understand the nature of the $\mathrm{Ca}^{2+}$ signal elevation during the pre-cataplexy episodes (except the AW episodes after predator odor exposure), we compared the $\mathrm{Ca}^{2+}$ signal intensities (average Z-scores) and peak frequencies between the Pre-C AW and the N-C AW, but we found no significant difference (Fig. 2F). Furthermore, we found that the sources or components of the Pre-C AWON cells and N-C AW-ON cells are very similar (Table 1 ). We did not find any significant difference between these two types of AW episodes.

\section{Discussion}

Cataplexy, presented as a sudden skeletal muscle paralysis or weakness during waking, is the most disabling symptom of sleep disorder narcolepsy (Reading, 2019). In murine models of narcolepsy, cataplexy usually occurs during the AW status of the animal, such as running, exploring, grooming, or responding to emotion stimuli (Overeem et al., 2001; Morawska et al., 2011; Reading, 2019). The brain circuitry responsible for cataplexy is not completely clear. Using the novel calcium imaging tool, we previously identified that the amygdala neuronal hyperactivity is associated with emotional cataplexy (Sun et al., 2019). In the present study, we recorded the in vivo activities of individual $\mathrm{MCH}$ neurons during cataplexy using the same methodology. We found that the majority of the recorded $\mathrm{MCH}$ neurons in the narcoleptic mice were most active during REM sleep, which is very similar to what occurs in wild-type mice (Blanco-Centurion et al., 2019; Izawa et al., 2019). Some recorded $\mathrm{MCH}$ neurons were also active during exploring, eating, and milk drinking, which is compatible with prior research about the effects of $\mathrm{MCH}$ neurons on food intake (Gonzalez et al., 2016; Dilsiz et al., 2020). Although prior studies indicated that $\mathrm{MCH}$ may regulate stress or fear (Carlini et al., 2006; Roy et al., 2006), we did not observe overt $\mathrm{MCH}$ neuron

Table 1: The numbers and percentages of on (activated) cells during the Pre-C AW were very similar to those during the NC AW

\begin{tabular}{lllll}
\hline & AW-ON only & REM-ON only & REMS-ON REMS/AW-ON & Unscored \\
N-C AW-ON cells (24) & $14(58.33 \%)$ & $0(0 \%)$ & $10(41.67 \%)$ & $0(0 \%)$ \\
Pre-C AW-ON cells (23) & $12(52.17 \%)$ & $1(4.35 \%)$ & $9(39.13 \%)$ & $1(4.35 \%)$ \\
\hline
\end{tabular}

No significant involvement of REM-ON only $\mathrm{MCH}$ neurons in Pre-C AW episodes was observed. 
activation during the innate fear coyote urine exposure, implicating that the $\mathrm{MCH}$ neurons that were usually active during $\mathrm{AW}$ were suppressed by fear in the narcoleptic mice. Further research on wild-type mice $\mathrm{MCH}$ neurons is need to explore the exact correlation between $\mathrm{MCH}$ neurons and fear regulation.

Nevertheless, our results demonstrated that $\mathrm{MCH}$ neurons always stayed inactive during both spontaneous and emotional cataplexy (induced by milk or coyote urine), indicating that $\mathrm{MCH}$ neuron activation is not necessary for maintaining cataplexy in orexin knock-out mice. Although $\mathrm{MCH}$ neuron activation was observed in some pre-cataplexy episodes, the activation intensity, pattern, and $\mathrm{ON}$ cell components were very similar to the activation noted in the regular AW episodes. In contrast to the amygdala GABAergic neurons that displayed abnormal hyperactivity and increased activated cell numbers during the pre-cataplexy episodes that were followed by the coyote urine-induced cataplexy (Sun et al., 2019), neither hyperactivity nor recruitment of more REM-ON MCH cells has been found in these pre-cataplexy $\mathrm{MCH}$ neuron activations. This implicates that the pre-cataplexy activations are nothing different from the activations during regular AW. Noticeably, some cataplexy bouts could happen even when the $\mathrm{MCH}$ neuron activation was absent (Fig. 2E). All this evidence indicates that $\mathrm{MCH}$ neuron activation during the pre-cataplexy episodes might be unnecessary for or irrelevant to cataplexy triggering. A previous study reported activated $\mathrm{MCH}$ neurons during cataplexy by examining c-Fos and $\mathrm{MCH}$ double-labeled neurons (Oishi et al., 2013). However, the c-Fos expression is an indirect marker of neuronal activation and lacks temporal and spatial specificities. There is a possibility that c-Fos expression induced by the AW behaviors immediately before cataplexy is mistaken as having been induced by cataplexy. Our work provides the first direct evidence of phenotype-specific and cataplexy-specific neuronal activities associated with $\mathrm{MCH}$ neurons.

Cataplexy is the most disabling feature in narcolepsy patients. Because of the similarities of the EEG spectrum and muscle atonia features, cataplexy has long been considered as a REM sleep intrusion into waking or abnormal REM sleep during waking (Roth et al., 1969; Luppi et al., 2011). Indeed, studies have found that cataplexy and REM sleep share some common neural substrates (Thakkar et al., 1999; Dauvilliers et al., 2014; Peever and Fuller, 2016). Thus, MCH neurons, an important REM sleep-regulating hub, are thought to trigger intrusions of REM sleep bouts into waking in the absence of orexin, consequently resulting in cataplexy. In support of this hypothesis, chemogenetic activation of $\mathrm{MCH}$ neurons increased cataplexy and abnormal REM sleep (Naganuma et al., 2018). However, some emerging evidence is not supportive of this hypothesis. For instance, stimulating cholinoceptor in the basal forebrain of narcoleptic dogs induced cataplexy attacks but did not block the cyclicity of burst of REM, implying that separate mechanisms underlie cataplexy and REM sleep (Nishino et al., 2000). $\mathrm{MCH}$ neuron activation is highly correlated with REM sleep in normal mice, while we found an explicit disassociation between $\mathrm{MCH}$ activation and cataplexy. The above evidence suggests that cataplexy and REM sleep might have discrete mechanisms and $\mathrm{MCH}$ neurons stay outside the cataplexy circuit. However, it does not necessarily exclude the possibility that $\mathrm{MCH}$ activation triggers abnormal REM sleep and muscle weakness. In other words, the abnormal REM sleep intrusion or muscle weakness induced by stimulating $\mathrm{MCH}$ neurons exogenously is mechanistically distinct from, albeit similar to, the muscle atonia seen in cataplexy of narcolepsy. Moreover, the silence of the $\mathrm{MCH}$ neurons during cataplexy implicates that their appropriate activation may inhibit cataplexy. Indeed, a recent study demonstrated that ablating both orexin and $\mathrm{MCH}$ neurons worsens cataplexy, revealing the possible role of $\mathrm{MCH}$ neurons in preventing cataplexy (Hung et al., 2019).

Notably, unlike in the orexin neuron ablation models for human narcolepsy (Hara et al., 2001; Tabuchi et al., 2014), the original orexin neurons in the orexin knock-out mice are still intact despite the loss of orexin peptides. These neurons might affect the activity of $\mathrm{MCH}$ neurons via other neuropeptides coexisting with orexin, such as dynorphin (Chou et al., 2001). Thus, a similar calcium imaging study on the orexin neuron ablation model is needed to further confirm our findings.

Sleep attack occurs in some narcoleptic patients and mice. In humans, it takes place when an overwhelming sense of sleepiness comes on quickly and is considered a symbol of excessive daytime sleepiness. In mice, such an attack is manifested as a short NREM sleep intrusion into waking (Hishikawa et al., 1968; Liu et al., 2011). It is probably caused by the increased sleep drive when orexin is missing, or when orexin neurons die. The missing $\mathrm{MCH}$ neuron activation supports the NREM sleep mechanism of sleep attacks.

In conclusion, we found that $\mathrm{MCH}$ neuron activation is unnecessary for the maintenance and initiation of cataplexy in orexin knock-out mice. Thus, $\mathrm{MCH}$ neurons might not be one of the crucial nodes in the cataplexy brain circuitry in orexin knock-out mice.

\section{Reference}

Adamantidis AR, Zhang F, Aravanis AM, Deisseroth K, de Lecea L (2007) Neural substrates of awakening probed with optogenetic control of hypocretin neurons. Nature 450:420-424.

Alexandre C, Andermann ML, Scammell TE (2013) Control of arousal by the orexin neurons. Curr Opin Neurobiol 23:752-759.

Blanco-Centurion C, Liu M, Konadhode RP, Zhang X, Pelluru D, van den Pol AN, Shiromani PJ (2016) Optogenetic activation of melanin-concentrating hormone neurons increases non-rapid eye movement and rapid eye movement sleep during the night in rats. Eur J Neurosci 44:2846-2857.

Blanco-Centurion C, Luo S, Spergel DJ, Vidal-Ortiz A, Oprisan SA, Van den Pol AN, Liu M, Shiromani PJ (2019) Dynamic network activation of hypothalamic MCH neurons in REM sleep and exploratory behavior. J Neurosci 39:4986-4998.

Carlini VP, Schiöth HB, de Barioglio SR (2006) Melanin-concentrating hormone $(\mathrm{MCH})$ reverts the behavioral effects induced by inescapable stress. Peptides 27:2300-2306.

Chemelli RM, Willie JT, Sinton CM, Elmquist JK, Scammell T, Lee C, Richardson JA, Williams SC, Xiong Y, Kisanuki Y, Fitch TE, Nakazato M, Hammer RE, Saper CB, Yanagisawa M (1999) 
Narcolepsy in orexin knockout mice: molecular genetics of sleep regulation. Cell 98:437-451.

Chou TC, Lee CE, Lu J, Elmquist JK, Hara J, Willie JT, Beuckmann CT, Chemelli RM, Sakurai T, Yanagisawa M, Saper CB, Scammell TE (2001) Orexin (hypocretin) neurons contain dynorphin. J Neurosci 21:RC168.

Dauvilliers Y, Siegel JM, Lopez R, Torontali ZA, Peever JH (2014) Cataplexy-clinical aspects, pathophysiology and management strategy. Nat Rev Neurol 10:386-395.

Dilsiz P, Aklan I, Sayar Atasoy N, Yavuz Y, Filiz G, Koksalar F, Ates T, Oncul M, Coban I, Ates Oz E, Cebecioglu U, Alp MI, Yilmaz B, Atasoy D (2020) MCH neuron dependent reward and feeding. Neuroendocrinology 110:258-270.

Gonzalez JA, lordanidou P, Strom M, Adamantidis A, Burdakov D (2016) Awake dynamics and brain-wide direct inputs of hypothalamic $\mathrm{MCH}$ and orexin networks. Nat Commun 7:11395.

Hara J, Beuckmann CT, Nambu T, Willie JT, Chemelli RM, Sinton CM, Sugiyama F, Yagami K, Goto K, Yanagisawa M, Sakurai T (2001) Genetic ablation of orexin neurons in mice results in narcolepsy, hypophagia, and obesity. Neuron 30:345-354.

Hishikawa Y, Nan'no H, Tachibana M, Furuya E, Koida H, Kaneko Z (1968) The nature of sleep attack and other symptoms of narcolepsy. Electroencephalogr Clin Neurophysiol 24:1-10.

Hof PR, Young WG, Bloom FE, Belichenko PV, Celio M (2000) Comparative cytoarchitectonic atlas of the C57BL/6 and 129/Sv mouse brains. New York: Elsevier.

Hung CJ, Ono D, Kilduff TS, Yamanaka A (2019) Dural orexin and $\mathrm{MCH}$ neuron-ablated mice display severe sleep attacks and cataplexy. BioRxiv 880229.

Hungs M, Mignot E (2001) Hypocretin/orexin, sleep and narcolepsy. Bioessays 23:397-408.

Izawa S, Chowdhury S, Miyazaki T, Mukai Y, Ono D, Inoue R, Ohmura Y, Mizoguchi H, Kimura K, Yoshioka M, Terao A, Kilduff TS, Yamanaka A (2019) REM sleep-active $\mathrm{MCH}$ neurons are involved in forgetting hippocampus-dependent memories. Science 365:1308-1313.

Jego S, Glasgow SD, Herrera CG, Ekstrand M, Reed SJ, Boyce R, Friedman J, Burdakov D, Adamantidis AR (2013) Optogenetic identification of a rapid eye movement sleep modulatory circuit in the hypothalamus. Nat Neurosci 16:1637-1643.

Konadhode RR, Pelluru D, Blanco-Centurion C, Zayachkivsky A, Liu M, Uhde T, Glen WB, van den Pol AN, Mulholland PJ, Shiromani PJ (2013) Optogenetic stimulation of $\mathrm{MCH}$ neurons increases sleep. J Neurosci 33:10257-10263.

Kirk RE (1968) Experimental design: procedures for the behavioral sciences. Belmont: Brooks/Cole.

Lin L, Faraco J, Li R, Kadotani H, Rogers W, Lin X, Qiu X, de Jong PJ, Nishino S, Mignot E (1999) The sleep disorder canine narcolepsy is caused by a mutation in the hypocretin (orexin) receptor 2 gene. Cell 98:365-376.

Liu M, Blanco-Centurion C, Konadhode R, Begum S, Pelluru D, Gerashchenko D, Sakurai T, Yanagisawa M, van den Pol AN, Shiromani PJ (2011) Orexin gene transfer into zona incerta neurons suppresses muscle paralysis in narcoleptic mice. $\mathrm{J}$ Neurosci 31:6028-6040.

Liu M, Blanco-Centurion C, Konadhode RR, Luan L, Shiromani PJ (2016) Orexin gene transfer into the amygdala suppresses both spontaneous and emotion-induced cataplexy in orexin-knockout mice. Eur J Neurosci 43:681-688.

Luppi PH, Clément O, Sapin E, Gervasoni D, Peyron C, Léger L, Salvert D, Fort P (2011) The neuronal network responsible for paradoxical sleep and its dysfunctions causing narcolepsy and rapid eye movement (REM) behavior disorder. Sleep Med Rev 15:153163.

Morawska M, Buchi M, Fendt M (2011) Narcoleptic episodes in orexin-deficient mice are increased by both attractive and aversive odors. Behav Brain Res 222:397-400.

Naganuma F, Bandaru SS, Absi G, Mahoney CE, Scammell TE, Vetrivelan R (2018) Melanin-concentrating hormone neurons contribute to dysregulation of rapid eye movement sleep in narcolepsy. Neurobiol Dis 120:12-20.

Nishino S, Riehl J, Hong J, Kwan M, Reid M, Mignot E (2000) Is narcolepsy a REM sleep disorder? Analysis of sleep abnormalities in narcoleptic Dobermans. Neurosci Res 38:437-446.

Oishi Y, Williams RH, Agostinelli L, Arrigoni E, Fuller PM, Mochizuki T, Saper CB, Scammell TE (2013) Role of the medial prefrontal cortex in cataplexy. J Neurosci 33:9743-9751.

Overeem S, Mignot E, van Dijk JG, Lammers GJ (2001) Narcolepsy: clinical features, new pathophysiologic insights, and future perspectives. J Clin Neurophysiol 18:78-105.

Peever J, Fuller PM (2016) Neuroscience: a distributed neural network controls REM sleep. Curr Biol 26:R34-R35.

Reading P (2019) Cataplexy. Pract Neurol 19:21-27.

Roth B, Brůhová S, Lehovsky“ M (1969) REM sleep and NREM sleep in narcolepsy and hypersomnia. Electroencephalogr Clin Neurophysiol 26:176-182.

Roy M, David NK, Danao JV, Baribault H, Tian H, Giorgetti M (2006) Genetic inactivation of melanin-concentrating hormone receptor subtype 1 (MCHR1) in mice exerts anxiolytic-like behavioral effects. Neuropsychopharmacology 31:112-120.

Scammell TE, Willie JT, Guilleminault C, Siegel JM; International Working Group on Rodent Models of Narcolepsy (2009) A consensus definition of cataplexy in mouse models of narcolepsy. Sleep 32:111-116.

Sun Y, Blanco-Centurion C, Bendell E, Vidal-Ortiz A, Luo S, Liu M (2019) Activity dynamics of amygdala GABAergic neurons during cataplexy of narcolepsy. Elife 8.

Tabuchi S, Tsunematsu T, Black SW, Tominaga M, Maruyama M, Takagi K, Minokoshi Y, Sakurai T, Kilduff TS, Yamanaka A (2014) Conditional ablation of orexin/hypocretin neurons: a new mouse model for the study of narcolepsy and orexin system function. $J$ Neurosci 34:6495-6509.

Thakkar MM, Ramesh V, Cape EG, Winston S, Strecker RE, McCarley RW (1999) REM sleep enhancement and behavioral cataplexy following orexin (hypocretin)-II receptor antisense perfusion in the pontine reticular formation. Sleep Res Online 2:112-120. 